\title{
Anoftalmia associada à catarata congênita: relato de caso
}

\author{
Anophthalmia and congenitalcataract:case report
}

\author{
AlessandroSantana ${ }^{1}$ \\ Karine Koller ${ }^{2}$ \\ Mauro Waiswol ${ }^{3}$
}

Trabalho realizado no Departamento de Oftalmologia da Santa Casa de Misericórdia de São Paulo (SP).

${ }^{1}$ Fellow do Serviço de Catarata da Santa Casa de Misericórdia de São Paulo. São Paulo (SP).

${ }^{2}$ Fellow do Serviço de Catarata da Santa Casa de Misericórdia de São Paulo. São Paulo (SP).

${ }^{3}$ Chefe do Serviço de Catarata da Santa Casa de Misericórdia de São Paulo e Doutor em Oftalmologia pela Universidade de São Paulo (USP). São Paulo (SP).

Endereço para correspondência: Alessandro Santana Rua Dona Veridiana, 107/74 - São Paulo (SP) CEP 01224-060

E-mail: alessandrosantana.oftalmo@ig.com.br

Recebido para publicação em 25.02.2004

Versão revisada recebida em 27.08.2004

Aprovação em 15.01.2005

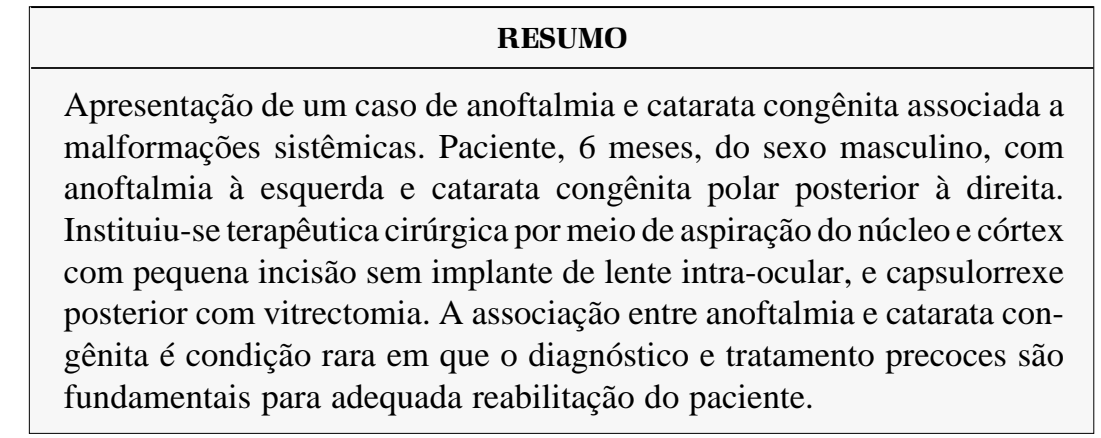

Descritores: Anoftalmia; Catarata/congênito; Órbita/anormalidades; Lactente; Masculino; Relatos de casos [Tipo de publicação]

\section{INTRODUÇ̃̃̃O}

Estima-se que existam 1,5 milhões de crianças cegas no mundo. Estes dados são baseados na melhor acuidade visual corrigida de $<20 / 400$. Um total de 1,3 milhões (cerca de $87 \%$ ) vivem em países em desenvolvimento ${ }^{(1)}$.

A etiologia da catarata congênita tem sido exaustivamente estudada, e as causas mais importantes são as aberrações genéticas, distúrbios metabólitos, prematuridade e infecções intra-útero ${ }^{(1-2)}$. A prevalência da catarata congênita é de 1 a 4/10.000 nascimentos em países industrializados e de 5 a 15/10.000 nascimentos em países em desenvolvimento, diferença justificada por ações de saúde pública e utilização da imunização nas causas infecciosas ${ }^{(2)}$. A causa hereditária é encontrada em 19,7\%, distúrbios metabólitos em $9 \%$, prematuridade em $9,2 \%$ dos casos de catarata congênita, e que $46 \%$ são de causa idiopática ${ }^{(3)}$.

Anomalias oculares podem ocorrer isoladas, em combinação, ou como parte de síndromes com malformações sistêmicas. Desta forma, a catarata congênita poderá fazer parte de um quadro sindrômico complexo, em que outros achados oculares ou sistêmicos possam estar associados ${ }^{(4)}$.

A anoftalmia é uma condição rara, caracterizada pelo crescimento orbitário deficiente, hipoplasia do globo ocular, além de fundo de saco conjuntival raso e alterações palpebrais ${ }^{(5)}$. Estudos epidemiológicos e genéticos, mostraram prevalência de 21/100.000 nascimentos. Anomalias sistêmicas estão presentes em $80 \%$ dos casos: malformações do sistema auditivo (47\%), sistema nervoso central $(42,5 \%)$, osteomuscular $(42,2 \%)$ e genital $(30,6 \%)^{(6)}$.

Matsui et al. demonstraram associação entre anoftalmia e catarata congênita $^{(7)}$.

O objetivo deste trabalho será apresentar um caso de catarata congênita com malformação orbitária e ocular associada a alterações sistêmicas. 


\section{RELATO DE CASO}

Paciente de sexo masculino, branco, 6 meses, procurou o Departamento de Oftalmologia da Santa Casa de São Paulo com história de malformação orbitária à esquerda (Figura 1).

$\mathrm{O}$ exame oftalmológico revelou olho esquerdo com órbita rasa, anoftalmia, fenda palpebral estreita com pequena abertura de $\pm 1 \mathrm{~mm}$, impossibilitando identificação das estruturas oculares (Figura 2). O exame biomicroscópico do OD apresentava córnea transparente, com diâmetro e espessura normais, câmara anterior formada, sem reação inflamatória, pupila redonda e central, catarata polar posterior $3+/ 4+$, com $4 \mathrm{~mm}$ de diâmetro. A oftalmoscopia direta e indireta, realizadas sob midríase, foram prejudicadas pela opacidade dos meios.

Realizou-se radiografia da face e ultra-sonografia ocular bilateral, mostrando olho direito com comprimento axial de aproximadamente $22 \mathrm{~mm}$ e cavidade vítrea normal (Figura 3 ).

Nascido de parto cesariano, com idade gestacional de 40 semanas e baixo peso (2,0 kg); apresentava palato "em ogiva", pé torto congênito e hérnia inguinal, corrigida no $2^{0}$ mês de vida (Figura 4).

Genitora, 18 anos, referia acompanhamento pré-natal bimestral, negava hipertensão arterial ou diabetes gestacional, apresentava sorologia negativa para sífilis, citomegalovírus e toxoplasmose, e imunização para rubéola com soro-conversão positiva. Pais sem consangüinidade.

O paciente foi submetido à cirurgia da catarata do olho direito (M.W.), utilizou-se a técnica de aspiração do núcleo e córtex com pequena incisão sem implante de lente intra-ocular, e capsulorrexe posterior com vitrectomia da hialóide e vítreo anterior.

O paciente evoluiu sem complicações no pós-operatório imediato (até 2 semanas), apresentando córnea transparente, câmara anterior formada sem reação inflamatória, pupila central e redonda, eixo visual livre. Foram prescritos colírios: dexametasona/neomicina/polimixina B $2 / 2 \mathrm{~h}$ e cicloplégico $8 / 8 \mathrm{~h}$, com re-

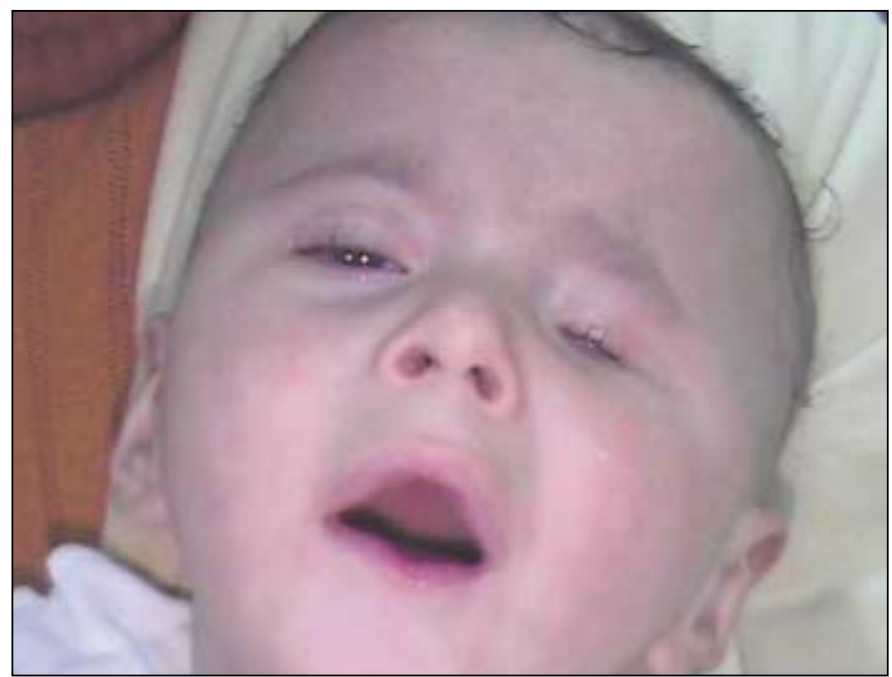

Figura 1 - Anoftalmia à esquerda

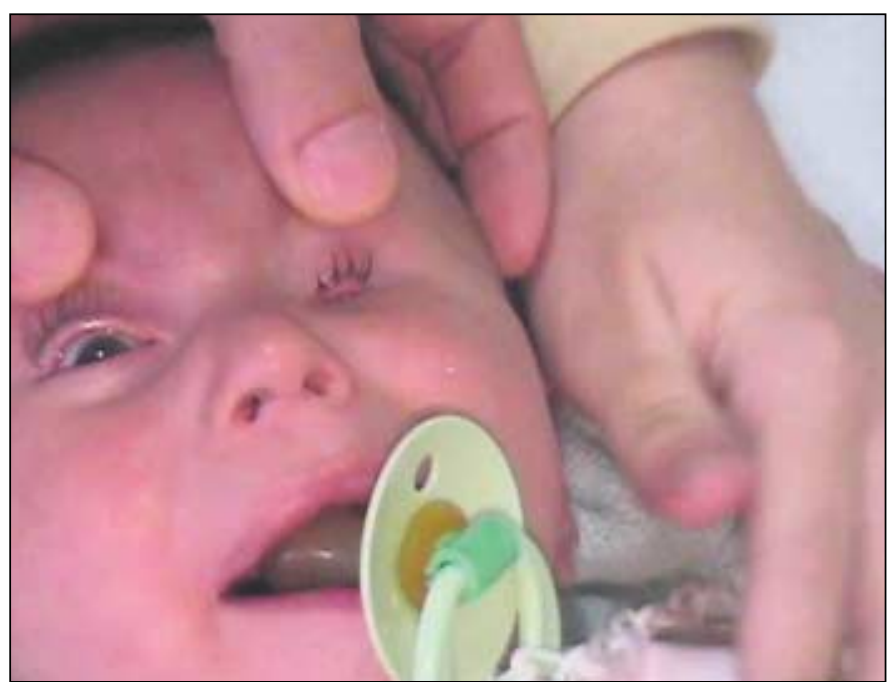

Figura 2 - Fenda palpebral estreita

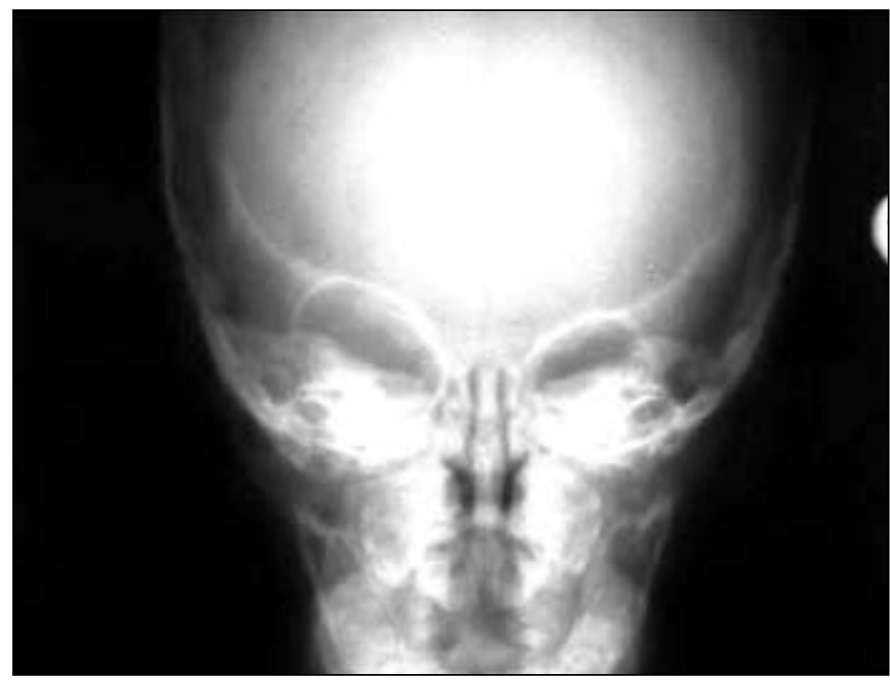

Figura 3 - Assimetria orbitária

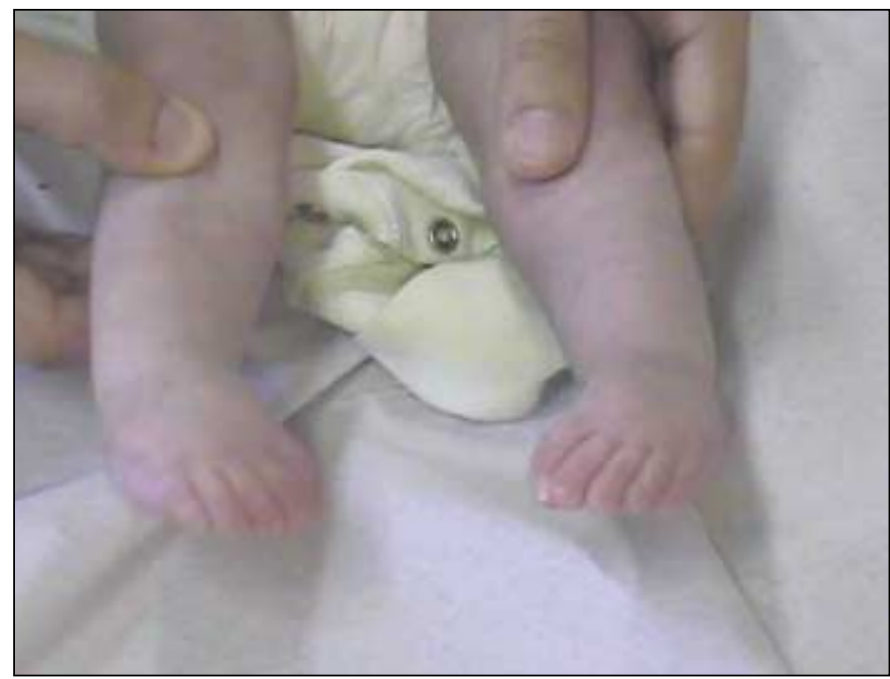

Figura 4 - Pé torto congênito 
dução lenta e gradual do corticóide. No $7^{0}$ dia de pós-operatório foi realizado exame sob narcose para cálculo refracional do paciente, sendo prescrito OD $+14,50$ dioptrias esféricas com adição de $+2,50$ esférico somado diretamente ao valor para longe, sem prescrição de bifocais, de acordo com Scott et al. ${ }^{(1)}$. À tonometria de aplanação apresentava $10 \mathrm{mmHg}$ e à oftalmoscopia direta, observava-se fundo róseo, papila com escavação fisiológica, mácula com reflexo foveal preservado, vasos de calibre e contornos regulares.

\section{DISCUSSÃO}

A anoftalmia é caracterizada por crescimento orbitário deficiente associado a hipoplasia do globo ocular, desta forma a correção da assimetria facial encontrada nestes casos, associada à identificação e tratamento de outras anomalias sistêmicas, é o ponto fundamental do tratamento ${ }^{(5)}$.

A terapêutica de escolha na anoftalmia é a utilização de expansores orbitários seriados. A intervenção em idade precoce poderá estimular o crescimento orbitário através da inserção inicial de expansores, utilizados desde as primeiras semanas de vida e substituídos a cada 3 a 6 semanas no primeiro ano de tratamento. Este tratamento visa à busca da melhor simetria facial e irá depender do grau de deformidade orbitária préexistente e da quantidade de conjuntiva residual ${ }^{(8-9)}$.

Nos casos severos, cirurgias para expansão do volume e transposição de músculos temporais são necessárias. Os resultados e a satisfação do paciente na maioria dos casos são pobres e a motivação e cooperação dos pais, são fatores importantes para o resultado final ${ }^{(9)}$.

No caso clínico descrito, optou-se pela utilização de próteses expansoras orbitárias com troca programada, inicialmente a cada 2 meses, para o tratamento da cavidade anoftálmica.

Estudos epidemiológicos demonstraram que existe uma estreita associação entre anoftalmia e outras malformações oculares e sistêmicas, necessitando do apoio de equipe multidisciplinar para condução destes casos, assim como a orientação e aconselhamento genético da família ${ }^{(6)}$. Busby et al. demonstrou que a anoftalmia é bilateral em $51 \%$ dos pacientes, com etiologia desconhecida na maioria dos $\operatorname{casos}^{(4)}$. Não apresenta predileção por sexo e maior freqüência entre gêmeos, além de um risco aumentado em idade materna avançada ${ }^{(10)}$.

A associação da anoftalmia com a catarata congênita é uma condição muito rara tendo sido sugerido a mesma relação patogenética na embriologia humana, supondo diversos mecanismos como desencadeadores da malformação ${ }^{(6)}$.

A importância do exame minucioso e adequado destes casos se faz necessário, especificamente no diagnóstico precoce da catarata congênita que associado a um tratamento da ambliopia no pós-operatório são os fatores vitais para o prognóstico visual destes olhos ${ }^{(2)}$.

A catarata congênita requer atenção para particularidades inerentes não encontradas na catarata senil, necessitando da disponibilidade e correta capacitação do cirurgião. As dificuldades variam desde a anatomia particular de olhos infantis, que apresentam cápsulas mais delgadas e elásticas, pressão vítrea positiva, maior índice de opacidade capsular e de hialóide anterior, inflamação pós-operatória e glaucoma secundário ${ }^{(11)}$.

O paciente apresentou uma incomum associação entre anoftalmia e catarata congênita, onde o diagnóstico precoce e pronta intervenção se tornaram relevantes em um paciente com olho único. Optou-se pela técnica de aspiração do núcleo e córtex através de capsulorrexe anterior. Realizou-se capsulorrexe posterior primária sem implante de lente intra-ocular, pois em pacientes infantis a opacificação de cápsula posterior no período pós-operatório tem apresentado elevada frequiência $^{(12)}$, além do fato do paciente se tratar de criança de 6 meses de idade, onde a capsulotomia com yag-laser se tornaria inviável sem sedação. A vitrectomia da hialóide anterior foi realizada com o objetivo de minimizar a inflamação e opacificação do eixo visual. A opção pela afacia à pseudofacia foi baseada em elevados índices de complicações relatadas na literatura, como: glaucoma, membrana pupilar inflamatória, corectopia e reoperações, relacionadas ao implante de lente intra-ocular em crianças de baixa idade ${ }^{(13-15)}$.

\section{CONCLUSÃO}

A estreita associação entre anoftalmia e outras malformações sistêmicas e oculares, incluindo a catarata congênita, indicam que o exame oftalmológico minucioso e uma visão global do paciente, através de equipe multidisciplinar é fundamental para uma adequada reabilitação do paciente.

\section{ABSTRACT}

The authors report a case of anophthalmia, congenital cataract and systemic malformations. Male patient, 6 months old, left anophthalmia and congenital posterior polar cataract in the right eye. The patient was treated with manual aspiration of the crystalline lens, with no intraocular lens implantation with primary posterior capsulorhexis and anterior vitrectomy through a small incision. The association of anophthalmia and congenital cataract is rare. The early diagnosis and management in these cases is very important for the best visual rehabilitation.

Keywords: Anophthalmos; Cataract/congenital; Orbit/abnormalities; Infant; Male; Case reports [Publication type]

\section{REFERÊNCIAS}

1. Lambert SR, Drack AV. Infantile cataracts. Surv Ophthalmol. 1996;40(6): 427-58.

2. Apple DJ, Assia EL. Pediatric cataract. Survey Ophthalmol. 2000;45(1):150-64.

3. Jain IS, Pillay P, Gangwar DN, Dhir SP, Kaul VK. Congenital cataract: etiology and morphology. J Pediatr Ophthalmol Strabismus. 1983;20(6):238-42.

4. Busby A, Dolk H, Collin R, Jones RB, Winter R. Compiling a national register of babies born with anophthalmia/microphthalmia in England 198894. Arch Dis Child Fetal Neonatal Ed. 1998;79(3):F168-73.

5. Krastinova D, Kelly MB, Mihaylova M. Surgical management of the anophthalmic orbit, part 1: congenital. Plast Reconstr Surg. 2001;108(4):817-26. 
6. Bermejo E, Martínez-Frias ML. Congenital eye malformations: clinical-epidemiological analysis of 1,124,654 consecutive births in Spain. Am J Med Genet. 1998;75(5):497-504.

7. Matsui H, Hayasaka S, Setogawa T. Congenital cataract in the right eye and primary clinical anophthalmos of the left eye in a patient with cerebellar hypoplasia. Ann Ophthalmol. 1993;25(8):315-8.

8. Sterker I, Klapper HU, Wiedemann P, Reiber T. [Clinical anophthalmos. Cosmetic outcome after 2 years therapy with an orbital expander for stimulating orbital growth]. Klin Monatsbl Augenheilkd. 2000;216(4):197-203. German.

9. Lee YH, Kim HC, Lee JS, Park WJ. Surgical reconstruction of the contracted orbit. Plast Reconstr Surg. 1999;103(4):1129-36; discussion 1137-8.

10. Källén B, Robert E, Harris J. The descriptive epidemiology of anophthalmia and microphthalmia. Int $\mathbf{J}$ Epidemiol. 1996;25(5):1009-16.
11. Katina JH, Lyra JMAG, Souza CD, Trindade FC. Tratamento cirúrgico da catarata pediátrica. Arq Bras Oftalmol. 2002;65(2):193.

12. Wilson ME. Management of aphakia in childhood. Focal Points. 1999;17(1): 1-16.

13. Koch DD, Kohnen T. Retrospective comparison of techniques to prevent secondary cataract formation after posterior chamber intraocular lens implantation in infants and children. J Cataract Refract Surg. 1997;23 Suppl 1:657-63.

14. Sharma N, Pushker N, Dada T, Vajpayee RB, Dada VK. Complications of pediatric cataract surgery and intraocular lens implantation. J Cataract Refract Surg. 1999;25(12):1585-8.

15. Lambert SR, Buckley EG, Plager DA, Medow NB, Wilson ME. Unilateral intraocular lens implantation during the first six months of life. J AAPOS. 1999;3(6):344-9. 